\title{
La canalisation sous-fluviale d'Arles à Trinquetaille
}

\author{
Par André COCHET *
}

L'étude des trente-trois tuyaux de plomb d'époque romaine conservés depuis 1822 au musée Réattu, provenant d'une canalisation immergée au fond du Rhône, longue d'environ $300 \mathrm{~m}$, pour conduire au faubourg de Trinquetaille l'eau de l'aqueduc d'Arles, révèle les précautions particulières prises pour mener à bien cette réalisation exceptionnelle. L'analyse des contraintes techniques d'un tel chantier permet d'évaluer les moyens mis en œuvre. L'existence d'un important lot de tuyaux (88 m de longueur conservée) permet d'estimer les tolérances de fabrication admises et les unités de longueur et de poids utilisées pour la conduite de ce chantier.

The sludy of about thirty big lead pipes of the Roman period, coming from a canalization immersed into the Rhone and conserved at the Reatlu Museum, reveals the particular precautions taken to build this exceptional realization. The canalization, approximatly $300 \mathrm{~m}$. long, was used to bring water to Trinquetaille's suburb originally coming from Arles' aqueduct. The existence of an important quantity of pipes ( $88 \mathrm{~m}$. length conserved) allow us to estimate the tolerance of making admitled and the units of length and weight used to lead this construction.

Mots clés : aqueduc, canalisation, génie-civil, tolérances (de fabrication), tuyaux de plomb, Arles, Bouchesdu-Rhône.

* CNRS, ERA 3, Maison de l'Orient, 7 rue Raulin, 69365 Lyon Cedex 07. 


\section{ÉTUDE TECHNIQUE}

Les parties conservées de la canalisation de plomb retirée du Rhône dans les circonstances rapportées en annexe I (p. 219) présentent un grand intérêt pour l'histoire des techniques.

Nous regrettons que rien ne nous soit parvenu des tuyaux de plomb plus anciennement retirés du Rhône par les ancres des bateaux, entre Arles et Trinquetaille, mais il faut cependant nous réjouir de la conservation de l'ensemble des éléments retirés du Rhône en 1822. Nous possédons ainsi plus de $88 \mathrm{~m}$ de la canalisation immergée au fond du fleuve, pour conduire à Trinquetaille de l'eau provenant de l'aqueduc d'Arles. C'est, pour la Gaule, le plus important témoin qui nous soit parvenu des canalisations de plomb largement utilisées par les Romains.

Les fragments conservés proviennent de toute évidence d'une conduite unique; ils présentent la même marque: C. CANTIVS POTHINVS. FAC, moulée avec la même plaque-modèle; ils constituent un lot de section homogène, et surtout, lors de la découverte, ils étaient solidaires les uns des autres, la plupart de leurs extrémités actuelles résultent soit d'un sciage postérieur à la découverte, pour faciliter la manutention, soit d'un déboîtement, par les efforts de traction nécessaires pour retirer du Rhône cette lourde conduite. La figure 1 montre un de ces fragments, les autres sont semblables, à l'exception des extrémités, les coupures modernes n'ayant pas toujours été situées de la même manière par rapport aux nœuds de soudure.

Si l'on fait abstraction d'une cassure moderne, au milieu d'un tuyau $\left(\mathrm{n}^{0} 10\right)$, dont les deux parties s'ajustent exactement nous n'avons que quatre extrémités résultant d'une cassure ou d'un écrasement.

Le musée Calvet d'Avignon possède deux fragments de la canalisation découverte en 1822 (CIL XII, 5701-2 h, numéros d'inventaire J471 et J472) qui lui furent offerts en 1834, par M. Montagnat. Leur étude a démontré qu'il s'agit de courts tronçons découpés de manière à conserver seulement la marque de C. Cantius Pothinus. Leurs dimensions transversales, l'épaisseur de leurs parois, les particularités de la soudure longitudinale attestent bien qu'ils faisaient partie de la même conduite que ceux conservés à Arles.

Le fragment de tuyau conservé au musée de Nîmes (fig. 2), porteur de la même marque (CIL XII, 5701-2 l), ne saurait provenir de la canalisation que nous étudions. S'il présente une section comparable

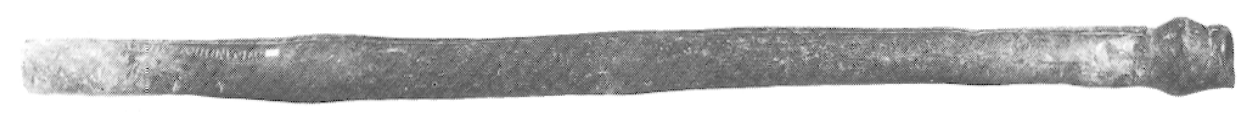

Fig. 1 - Fragment $\mathrm{n}^{0} 1$ (longueur : $281 \mathrm{~cm}$ ). Les deux extrémités correspondent à des découpages modernes (sciages). A gauche, marque du fabricant; à droite, noud de soudure et petit fragment d'un second élément de la canalisation.

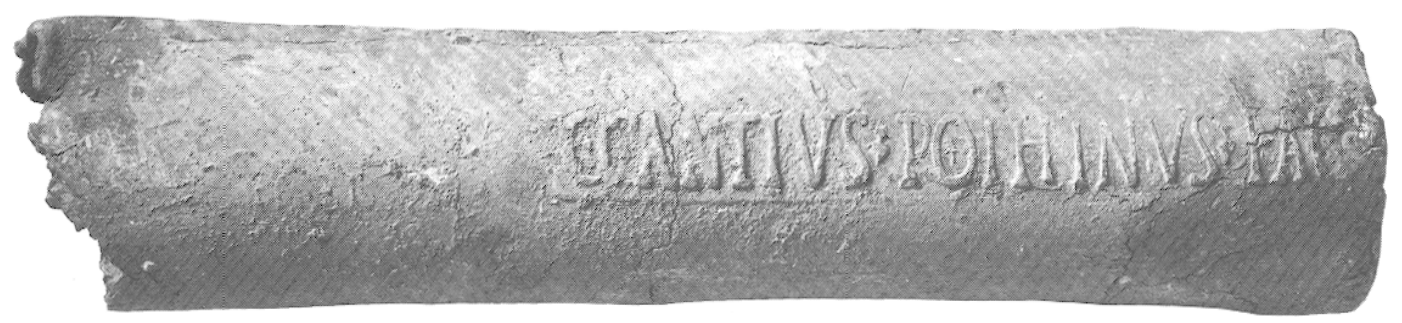

Fig. 2 - Fragment de tuyau marqué C.CA.VTIVS.POTHINVS.FAC. conservé au musée de Nîmes. Il n'y a pas de cordon de soudure saillant (soudure type II). Autour de l'inscription, on voit le contour de la petite "plaque-modèle" servant à imprimer l'inscription en creux sur le sable du moule. Dimensions : longueur $57 \mathrm{~cm}$; périmètre extérieur $32 \mathrm{~cm}$; longueur de l'inscription $32,5 \mathrm{~cm}$; hauteur des lettres 2,8 à $3 \mathrm{~cm}$. 
ses parois sont bien plus minces et sa soudure longitudinale est d'un type différent ${ }^{1}$. Son existence nous prouve que C. Cantius Pothinus a fourni d'autres chantiers que celui de la traversée du Rhône.

Les découvertes perdues provenaient-elles de la même canalisation, ou bien nous auraient-elles fait connaître l'existence d'une autre conduite parallèle à celle que nous avons pu étudier, comme leur abondance pourrait le faire supposer? La question ne peut être tranchée de manière catégorique, les quelques indications qui sont conservées (marque $\mathrm{C}$. CANTIVS. POTHINVS. FAC, dimensions des éléments) suggéreraient cependant que la trouvaille de 1707 faisait partie du même ensemble que celle de 1822.

\section{DESCRIPTION}

La conduite a été réalisée en assemblant bout à bout des éléments d'environ $3 \mathrm{~m}$ de long.

Aucun repère n'a été disposé lorsque la canalisation a été découpée pour permettre son transport au Musée, les faces résultant du sciage de tuyaux de section presque semblable ne fournissent aucun raccord certain pour déterminer l'ordre dans lequel se trouvaient les fragments conservés, nous ne pouvons donc pas reconstituer le puzzle. Le nombre des éléments représentés demeure même incertain : au moins 31 , au plus 33 , les plus petits des fragments pouvant provenir d'un même tuyau de 10 pieds ou de tuyaux différents.

Les coupures faites en 1822 se situent au voisinage des assemblages, ou même dans les assemblages si bien que la plupart des fragments actuels correspondent à la plus grande partie ou à la totalité d'un élément antique.

Le tableau I donne les dimensions de ces fragments, les dessins (fig. 3 et 4 ) donnent la signification des cotes portées dans les premières colonnes de ce tableau.

1 Au musée de Nîmes, sans numéro d'inventaire (fonds ancien). A. Pelet (catalogue du musée de Nîmes, 1853, p. 147, $n^{\circ} 193$ ) indique : "Découvert en 1742, à la Fontaine. M. Noble de la Lauzière le rapporte sous le $\mathrm{n}^{\circ} 17$ comme étant à Arles». Les dimensions sont indiquées dans la légende de la figure 2 .

La soudure est du type II défini dans A. Cochet, J. HANSEN, Conduites et objets de plomb gallo-romains de Vienne, $46^{\mathrm{e}}$ suppl. à Gallia, 1986, p. 28-34.
Des mesures relevées nous avons déduit le poids du tuyau, en retirant du résultat de notre pesée le poids estimé du métal de la soudure employé pour les assemblages (en tablant sur la moyenne des dimensions du nœud de soudure, sur une densité de 10 pour l'alliage plomb-étain, et en comptant pour chaque fragment, selon la position des coupes effectuées au siècle dernier, de zéro à deux nœuds de soudure (tabl. I, colonne K : "poids corrigé»). De ce poids corrigé découle l'évaluation du poids par mètre des tuyaux employés, et celle de l'épaisseur moyenne des feuilles de plomb, obtenue en calculant la surface de la section assimilée à une couronne circulaire surmontée d'un rectangle (cordon de soudure).

Ce calcul est nécessaire car la mesure directe de l'épaisseur n'est possible qu'au voisinage des extrémités des fragments, sur une portion de quelques centimètres, lorsque cette extrémité ne coïncide pas avec un emboîtement de deux éléments : cette mesure peut ne pas être représentative de l'épaisseur moyenne d'un élément.

Chaque élément a été façonné en cintrant une feuille plane, de manière à rapprocher les deux bords opposés, réunis par une soudure à l'aide de plomb fondu, coulé sur l'assemblage, formant un cordon rectangulaire sur la génératrice supérieure du tuyau ${ }^{2}$.

La marque C. CANTIVS. POTHINVS. FAC est venue de fonderic lors de la couléc des feuilles de plomb. Étudiée dans l'annexe II (p. 220), elle nous donne le nom de l'artisan qui a façonné les tuyaux (ou les a fait fabriquer par ses ouvriers). En outre, huit éléments portent sur la face plane du cordon de soudure longitudinal, une inscription numérique, incisée à l'aide d'un burin pour les segments rectilignes des lettres X I L et d'une gouge pour l'arc de cercle du C (fig. 5). Ces inscriptions numériques incisées sont communément interprétées comme l'indication du poids de l'élément marqué, nous reviendrons plus loin sur ce sujet.

Les éléments sont reliés entre eux par un emboîtement de 3 à $4 \mathrm{~cm}$ de long, recouvert d'un nœud de soudure (jonction "en olive» des plombiers modernes) formé d'un alliage plomb-étain.

Chacun de ces points mérite une analyse détaillée, que nous présentons en comparant la canalisation d'Arles aux éléments conservés d'une canalisa-

2 Pour la description détaillée du processus de fabrication cf. ibid., p. 29 ; il s'agit du type ID. 


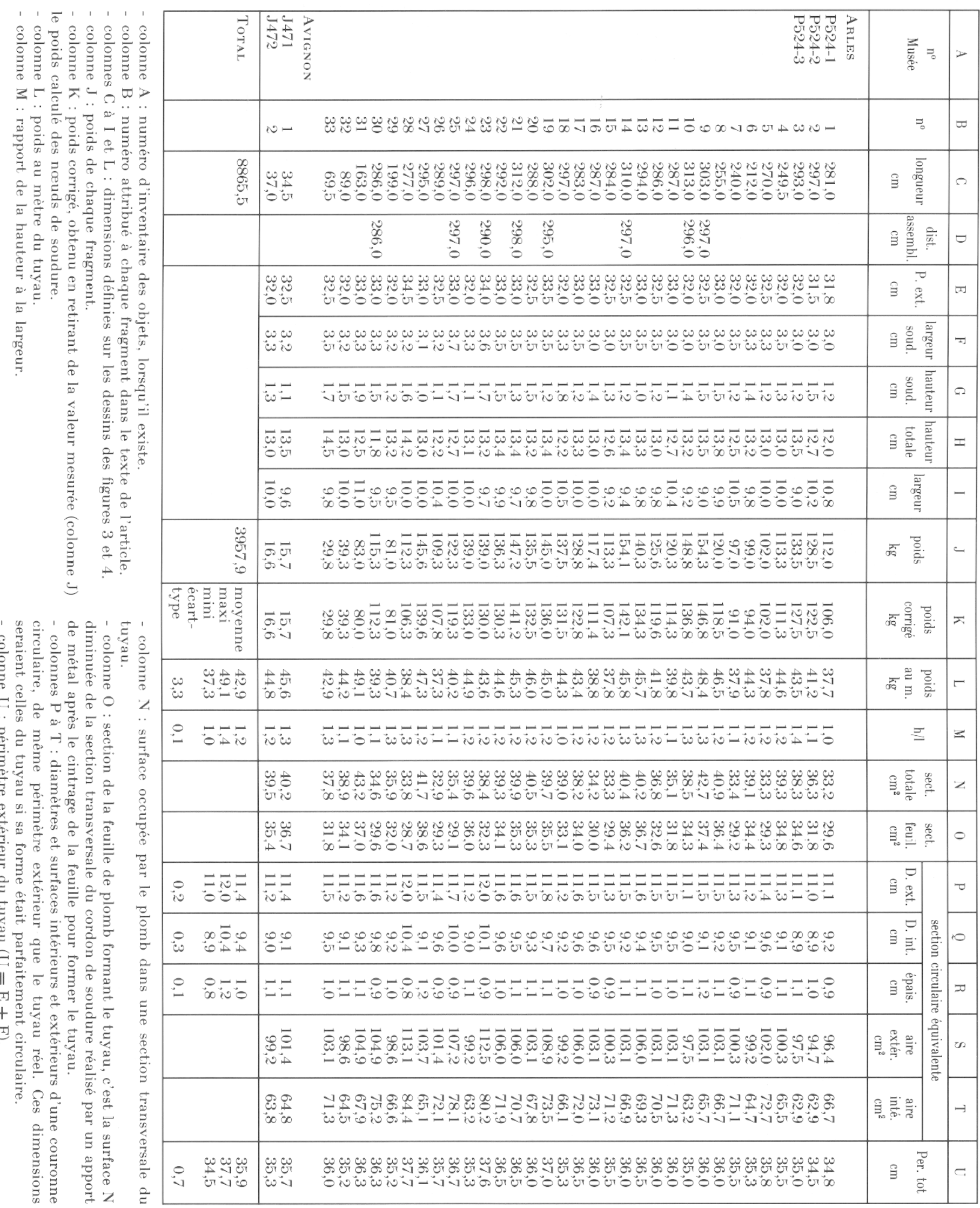




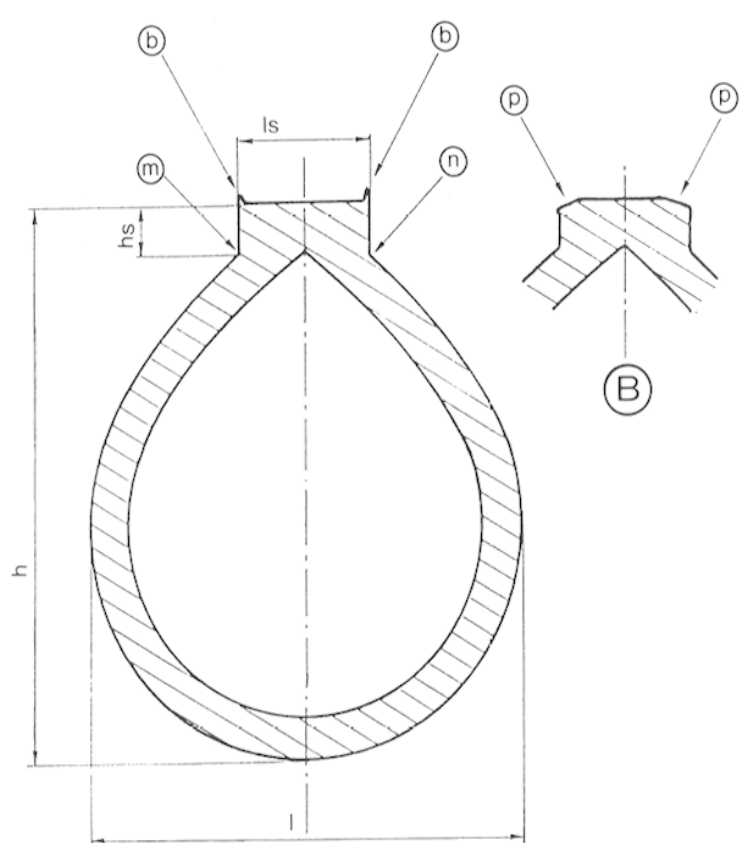

(A) $\leftarrow$ Fig. 3 - Section transversale de la canalisation; définition des cotes utilisées dans le tableau I : $\mathrm{h}$ : hauteur totale de la section (colonne $\mathrm{H}$ ); $\mathrm{l}$ : largeur totale de la section (colonne I); hs : hauteur du cordon de soudure (colonne G); Is : largeur du cordon de soudure (colonne $F$ ) : le périmètre extérieur (colonne E) est mesuré selon l'arc m-n.

A. En b-b, bavures verticales encadrant par places le cordon de soudure.

B. En p-p, petits plats formés par le martelage qui, par places, ont fait disparaitre ces bavures.

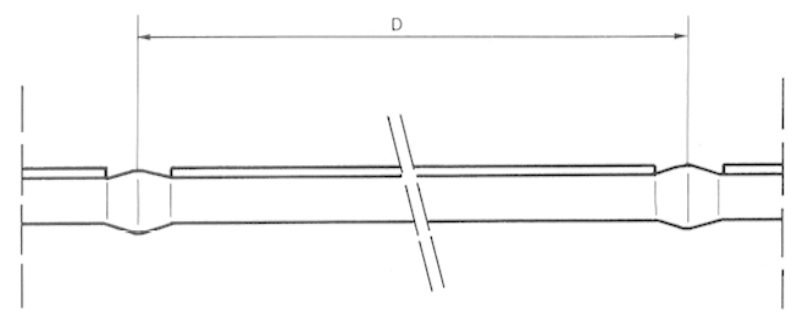

Fig. 4 - Distance entre les nœuds de soudure, mesurée entre les parties les plus épaisses de ces nœuds (tabl. I, col. D).

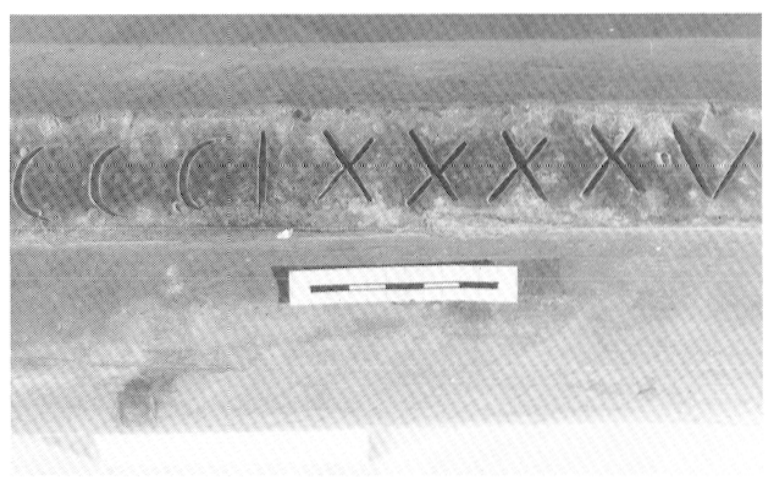

Fig. 5 - Inscription incisée sur la face supérieure du cordon de soudure du fragment $n^{\circ} 12$.

Lyon : $1,2 \mathrm{~cm}$ contre $0,6 \mathrm{~cm}$. Les nombreux tuyaux que nous avons étudiés dans diverses villes sont tous, à section voisine, bien plus minces que ceux retirés du Rhône. L'épaisseur de $1 \mathrm{~cm}$ n'étant atteinte ou dépassée que pour des tuyaux de périmètre extérieur supérieur à $50 \mathrm{~cm}$.

\section{CINTRAGE DES FEUILLES}

Il est difficile de plaquer exactement sur un mandrin cylindrique les bords d'une feuille plane (fig. 6). Pour arriver à ce résultat il faudrait exercer une pression considérable. C. Cantius Pothinus a 
Tableau II - Dimensions des fragments conservés :

\begin{tabular}{|c|c|c|c|c|c|c|c|c|c|c|}
\hline A & $\mathrm{B}$ & $\mathrm{G}$ & $\mathrm{D}$ & $\mathrm{E}$ & $\mathrm{F}$ & $\mathrm{G}$ & $\mathrm{H}$ & $\mathrm{I}$ & $\mathrm{J}$ & $\mathrm{K}$ \\
\hline $\begin{array}{c}\mathrm{n}^{\circ} \\
\text { Musée }\end{array}$ & \multirow{2}{\mathrm{n}^{0}}{} & $\begin{array}{c}\text { longueur } \\
\mathrm{cm}\end{array}$ & $\begin{array}{c}\text { dist. } \\
\text { assembl. } \\
\mathrm{cm}\end{array}$ & $\begin{array}{c}\text { P. ext. } \\
\mathrm{cm}\end{array}$ & $\begin{array}{c}\text { largeur } \\
\text { soud. } \\
\mathrm{cm}\end{array}$ & $\begin{array}{c}\text { hauteur } \\
\text { soud. } \\
\mathrm{cm}\end{array}$ & $\begin{array}{c}\text { hauteur } \\
\text { totale } \\
\mathrm{cm}\end{array}$ & $\begin{array}{c}\text { largeur } \\
\mathrm{cm}\end{array}$ & $\begin{array}{c}\text { poids } \\
\mathrm{kg}\end{array}$ & $\begin{array}{c}\text { poids } \\
\text { corrigé } \\
\mathrm{kg}\end{array}$ \\
\hline LYoN & & & & & & & & & & \\
PB25 & & 172,0 & & 32,8 & 3,0 & 1,0 & 13,0 & 11,5 & 48,1 & 45,5 \\
PB24 & & 130,0 & & 33,0 & 3,5 & 0,9 & 13,0 & 11,5 & 37,0 & 36,3 \\
PB23 & & 231,5 & & 33,2 & 3,0 & 0,9 & 11,5 & 9,5 & 63,5 & 62,8 \\
\hline PB26 & & 57,5 & & 33,0 & 3,0 & 0,9 & 11,0 & 10,0 & 15,1 & 15,4 \\
\hline
\end{tabular}

\begin{tabular}{|c|c|c|c|c|c|c|c|c|c|c|}
\hline A & $\mathrm{L}$ & M & $\mathrm{N}$ & 0 & $\mathrm{P}$ & Q & $\mathrm{R}$ & $\mathrm{s}$ & $\mathrm{T}$ & $\mathrm{U}$ \\
\hline \multirow{2}{*}{$\begin{array}{c}\mathrm{n}^{0} \\
\text { Musée }\end{array}$} & \multirow{2}{*}{$\begin{array}{c}\text { poids } \\
\text { au m. } \\
\text { kg }\end{array}$} & \multirow{2}{*}{$\mathrm{h} / \mathrm{l}$} & \multirow{2}{*}{$\begin{array}{c}\text { sect. } \\
\text { totale } \\
\mathrm{cm}^{2}\end{array}$} & \multirow{2}{*}{$\begin{array}{l}\text { sect. } \\
\text { feuil. } \\
\mathrm{cm}^{2}\end{array}$} & \multicolumn{5}{|c|}{ section circulaire équivalente } & \multirow{2}{*}{$\begin{array}{c}\text { Per. tot } \\
\mathrm{cm}\end{array}$} \\
\hline & & & & & $\begin{array}{c}\text { D. ext. } \\
\mathrm{cm}\end{array}$ & $\begin{array}{c}\text { D. int. } \\
\mathrm{cm}\end{array}$ & $\begin{array}{c}\text { épais. } \\
\mathrm{cm}\end{array}$ & $\begin{array}{c}\text { aire } \\
\text { extér. }\end{array}$ & $\begin{array}{l}\text { aire } \\
\text { inté. }\end{array}$ & \\
\hline LYON & & & & & & & & & & \\
\hline PB25 & 26,5 & 1,0 & 23,3 & 20,3 & 11,4 & 10,2 & 0,6 & 102,0 & 81,7 & 35,8 \\
\hline PB24 & 27,9 & 1,1 & 24,6 & 21,5 & 11,6 & 10,4 & 0,6 & 106,0 & 84,6 & 36,5 \\
\hline PB23 & 27,1 & 1,1 & 23,9 & 21,2 & 11,5 & 10,3 & 0,6 & 104,3 & 83,1 & 36,2 \\
\hline Moyenne & 27,2 & 1,1 & & & & & 0,6 & & & 36,2 \\
\hline PB26 & 26,8 & 1,0 & 23,6 & 20,9 & 11,5 & 10,2 & 0,6 & 103,1 & 82,2 & 36 \\
\hline
\end{tabular}

- colonne A : numéro d'inventaire des objets, lorsqu'il existe. - colonne $\mathrm{B}$ : numéro attribué à chaque fragment dans le texte de l'article.

- colonne C à I et L : dimensions définies sur les dessins des figures 3 et 4 .

- colonne J : poids de chaque fragment.

- colonne K : poids corrigé, obtenu en retirant de la valeur mesurée (colonne J) le poids calculé des nouds de soudure.

- colonne L : poids au mètre du tuyau.

- colonne M : rapport de la hauteur à la largeur.
- colonne N : surface occupée par le plomb dans une section transversale du tuyau.

- colonne 0 : section de la feuille de plomb formant le tuyau, c'est la surface $\mathrm{N}$ diminuée de la section transversale du cordon de soudure réalisé par un apport de métal après le cintrage de la feuille pour former le tuyau.

- colonnes P à T : diamètres et surfaces intérieurs et extérieurs d'une couronne circulaire, de mème périmètre extérieur que le tuyau réel. Ces dimensions seraient celles du tuyau si sa forme était parfaitement circulaire.

- colonne U : périmètre extérieur du tuyau $(\mathrm{U}=\mathrm{E}+\mathrm{F})$.

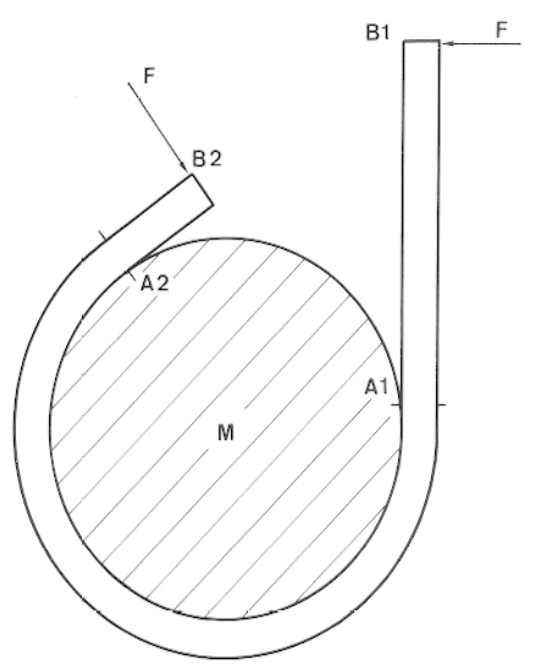

Fig. 6 - Un cintrage parfait est impossible sur un mandrin cylindrique; au fur et à mesure que la feuille de plomb s'enroule sur le mandrin $\mathrm{M}$ le bras de levier $\mathrm{AB}$ avec lequel agit la force $\mathrm{F}$, appliquée sur le bord de la feuille, va en décroissant (A2B2<A1B1). Il subsiste donc deux petits pans peu ou pas courbés au voisinage de la soudure des tuyaux. 
donné à ses tuyaux une forme un peu plus haute que celle réalisée à Lyon, le rapport de la hauteur à la largeur de la section étant en moyenne de 1,18 contre 1,07 .

Ccla pourrait être dû à la grande épaisseur du métal, rendant le cintrage malaisé. Il faut cependant observer que la forme la plus proche du cylindre n'a pas été obtenue à Lyon par la seule opération de cintrage; après l'opération de soudure, un vigoureux martelage des deux arêtes longitudinales du cordon de soudure a contribué à diminuer la hauteur du tuyau et à accroître sa largeur pour lui donner une section se rapprochant davantage d'un cercle ${ }^{4}$. Le martelage qu'on observe sur une partie de la longueur de la canalisation d'Arles semble avoir été beaucoup plus léger, se bornant à écraser des bavures formées le long de la soudure longitudinale qui ferme les tuyaux.

\section{SOUDURE LONGITUDINALE}

La soudure longitudinale qui ferme les tuyaux présente une section générale rectangulaire, les colonnes $\mathrm{F}$ et $\mathrm{G}$ du tableau I donnent les dimensions moyennes pour chaque fragment. En coupe, ce cordon se présente de deux manières différentes (fig. 7), par places (fig. 3A) les deux faces latérales, lisses, sont prolongées par deux bavures verticales, encadrant une face supérieure horizontale, plane et lisse en dehors de quelques défauts de coulée. En d'autres endroits, deux petits pans coupés obliques remplacent les bavures (fig. 3B). Ce cordon présente aussi de brusques changements de hauteur (fig. 9) qui marquent des reprises dans l'exécution.

Le métal d'apport a été coulé entre deux règles et, avant sa solidification, on a appuyé sur sa surface avec un instrument plan, sans doute dans l'intention de le forcer à pénétrer entre les deux bords de la feuille à souder (dispositif représenté fig. 8). Un léger martelage a fait, par places, disparaître ces bavures qui sont alors remplacées par de petits pans coupés formés par l'écr'dsement du métal.

Cette soudure diffère de celle des tuyaux lyonnais, qui présente une hauteur uniforme tout au long de chaque section, et un martelage beaucoup plus prononcé sur les côtés du cordon.

Il semble bien que C. Cantius Pothinus ait dû exécuter cette soudure par petites longueurs; le métal se solidifiant assez rapidement, l'opération de

4 Il s'agit du type IB' $^{\prime}$ défini dans A. Cochet, J. HAN$\mathrm{SEN}$, Conduiles et objels de plomb gallo-romains de Vienne, $46^{\mathrm{e}}$ suppl. à Gallia, 1986, p. 29.

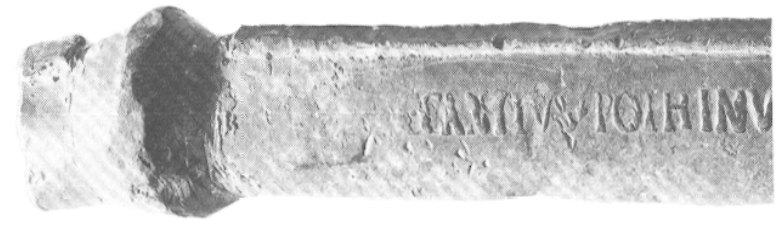

Fig. 7 - Un nœud de soudure : les cordons de soudure ont été enlevés pour l'assemblage. Le cordon présente deux aspects, avec et sans martelage.

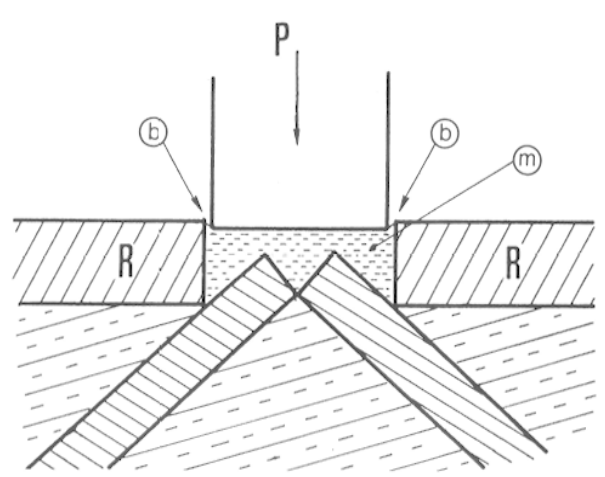

Fig. 8 - Dispositif pour l'exécution de la soudure longitudinale. Le métal d'apport $m$ est versé entre les deux règles $R$ (reliées entre elles pour les empècher de s'écarter) puis un outil $P$ exerce une pression sur la surface libre du métal d'apport; en b-b : bavures.

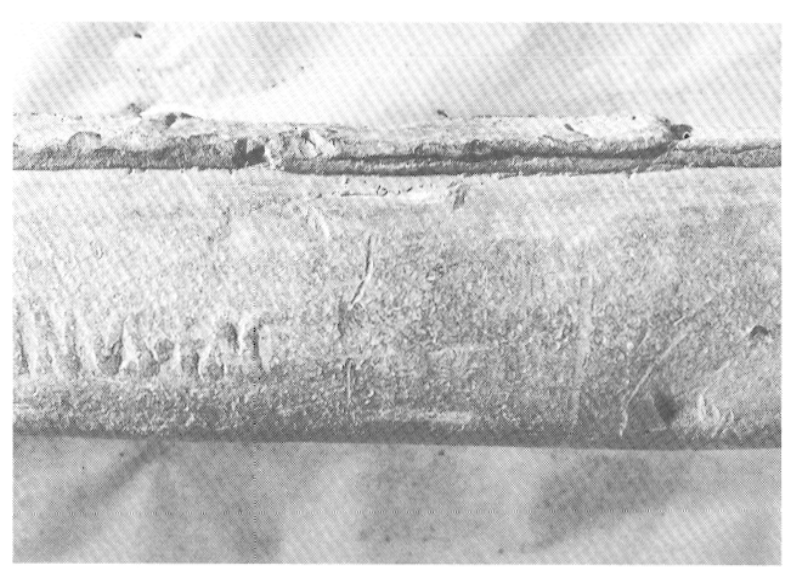

Fig. 9 - Reprise dans l'exécution de la soudure longitudinale.

refoulement ne peut être conduite que par petites étapes, pendant le temps assez court durant lequel le métal d'apport demeure liquide (fig. 9).

En dépit de cette technique particulière, que nous n'avons rencontrée encore nulle part ailleurs, la qualite de la soudure n'est pas toujours parfaite. Il arrive que le cordon adhère mal au métal de la feuille, à la base de ses faces latérales. En d'autres 


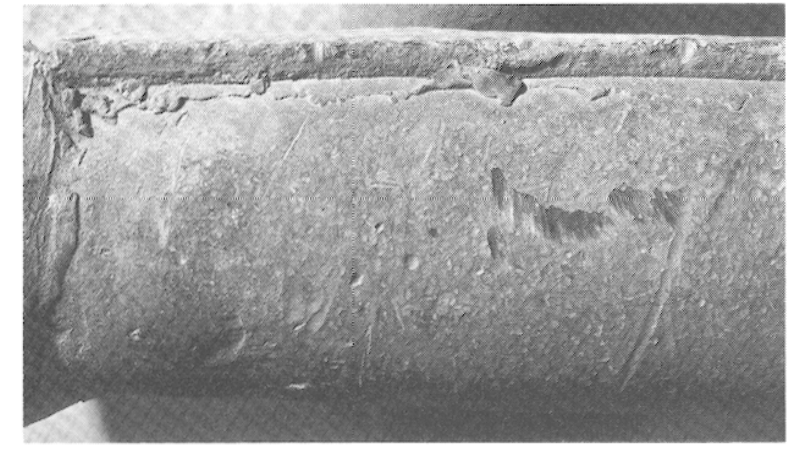

Fig. 10 - A gauche, bavures à la base du cordon de soudure; à droite, cordon adhérant imparfaitement à la feuille formant le tuyau.

points, le métal d'apport a coulé entre la feuille de plomb et la règle délimitant le cordon de soudure désiré, formant des bavures (fig. 10). Un tuyau $\left(n^{\circ} 17\right)$ présente même une soudure qui a fait l'objet d'une réparation, par une coulée supplémentaire de métal liquide, formant un second cordon à côté du premier.

\section{ASSEMBLAGE DES ÉLÉMENTS ENTRE EUX}

L'assemblage se fait par un emboittement assez court (3 à $4 \mathrm{~cm}$ ), l'extrémité d'un des tuyaux étant un peu évasée pour recevoir celle de l'autre tuyau, débarrassé sur quelques centimètres de son cordon de soudure (fig. 11). Dans la plupart des assemblages conservés, un ou deux gros clous de fer transpercent cet emboîtement. Il s'agit de clous à tige carrée, longs de $12 \mathrm{~cm}$ environ, qui traversent ainsi entièrement l'espace intérieur du tuyau, jusqu'au contact de la paroi, à l'opposé de leur point d'entrée (fig. 12 et 13). Ils jouent ainsi le rôle d'une goupille s'opposant au déboîtement de l'assemblage par traction. Cette particularité avait déjà été remarquée par J.-M. Rouquette dans son mémoire de diplôme d'études supérieures, en 1954. Nous ne connaissons aucun autre exemple d'emploi de tels clous transversaux. Le tout est recouvert d'une masse de soudure (alliage plomb-étain) formant deux troncs de cônes opposés par leurs grandes bases («jonction en olive»). Les dimensions de cette masse de soudure varient peu : longueur de 12 à $14 \mathrm{~cm}$, périmètre maximal de 51 à $53 \mathrm{~cm}$.

A Lyon, on observe le même type d'emboîtement (procédé extrêmement répandu), mais sanıs les clous transversaux et, d'après la soudure restée

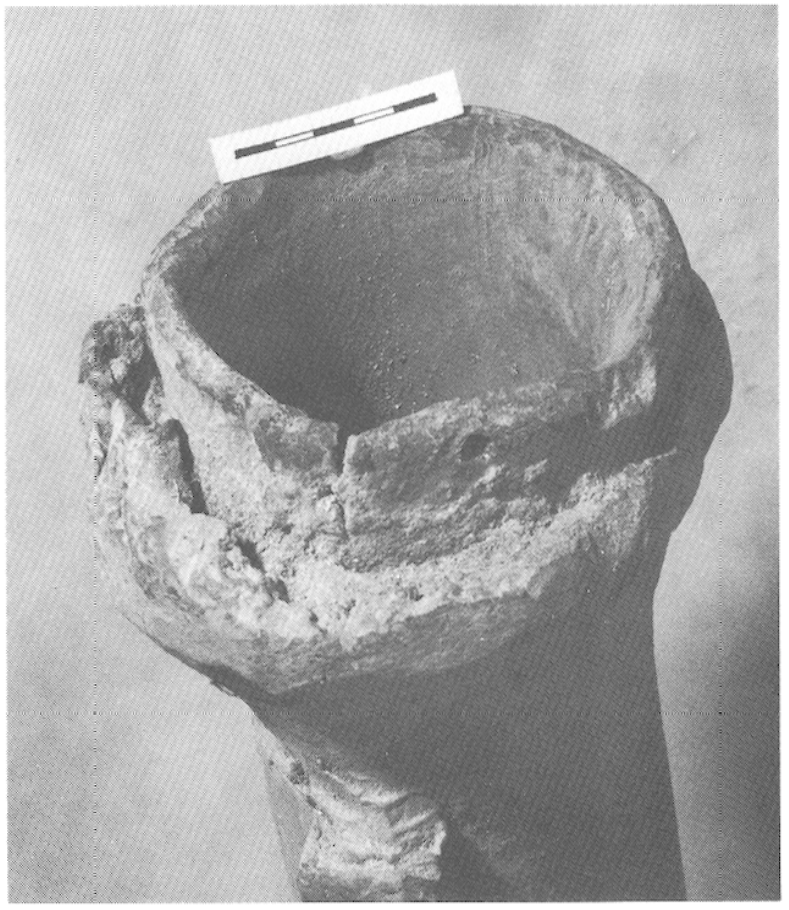

Fig. $11-\Lambda$ ssemblage déboîté, le trou atteste la présence d'un clou transversal disparu.

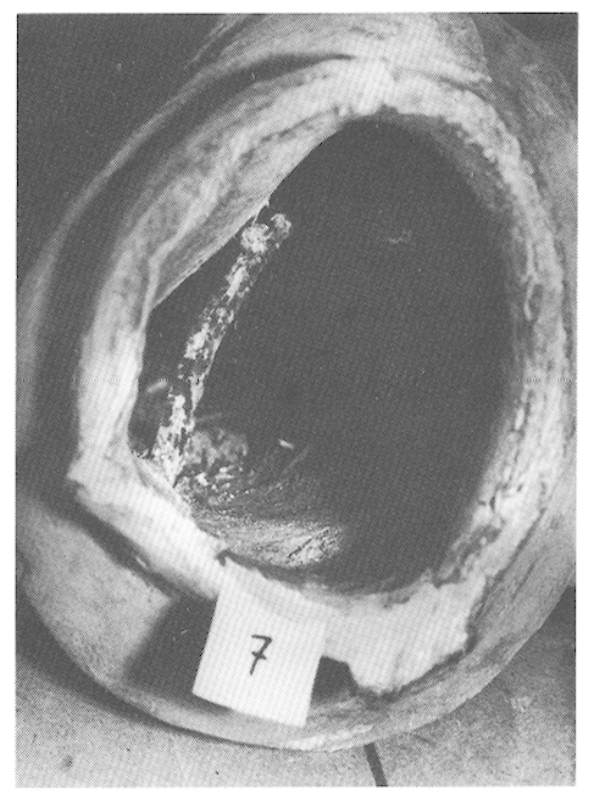

Irig. 12 - Clou transversal dans un emboitement (élément $\mathrm{n}^{\circ} 7$ ). 


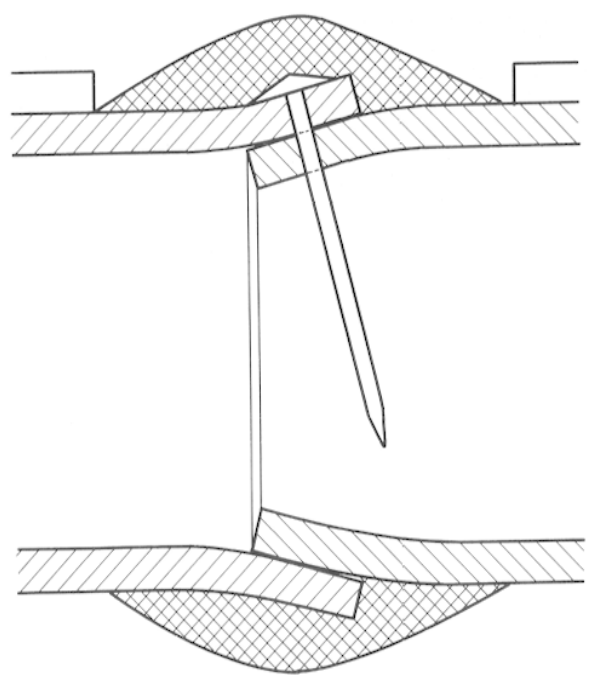

Fig. 13 - Coupe longitudinale d'un assemblage de la conduite d'Arles avec clou traversant l'assemblage.

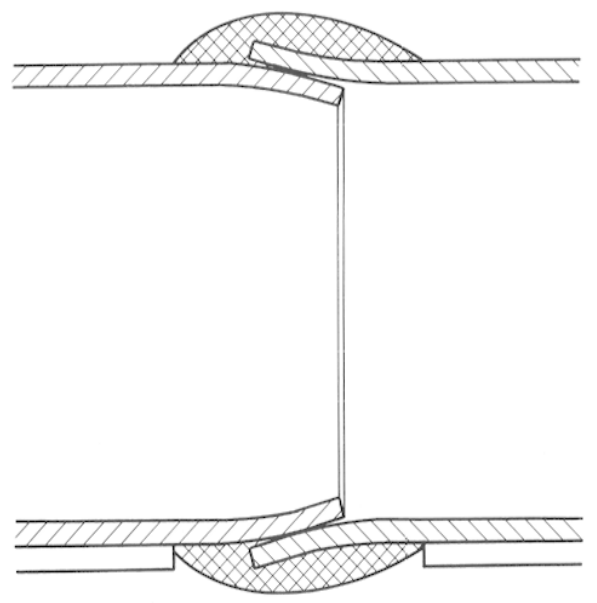

Fig. 14 - Coupe longitudinale d'un assemblage de la conduite de Lyon.

adhérente aux divers tuyaux (tous les assemblages sont rompus) la longueur totale du nœud de soudure ne dépassait guère $7 \mathrm{~cm}$ et son périmètre maximal $40 \mathrm{~cm}$, sur des emboîtements de $3,5 \mathrm{~cm}$ (fig. 14).

\section{INTERPRÉTATION DES PARTICULARITÉS}

La grande épaisseur des parois, la recherche d'une soudure longitudinale solide, à laquelle on a évité l'épreuve d'un martelage poussé, la recherche d'assemblages inler-éléments aussi résistants que possible montrent que le constructeur a accordé la priorité à la robustesse de l'installation, au détriment de son débit. En effet, une section circulaire offre la plus grande surface de passage à l'eau, pour un périmètre donné mais le maître d'œuvre a accepté de s'en écarter un peu pour éviter le risque d'abîmer la soudure; avec les clous traversant la veine liquide, il a introduit dans la canalisation des éléments générateurs de tourbillons et de pertes de charge.

Ce choix de la robustesse avec le surcoût qu'il entraîne est justifié pour une conduite destinée à être immergée dans un fleuve à courant rapide où elle devient inaccessible, donc irréparable, après la pose au cours de laquelle elle est soumise à des efforts importants.

Le résultat semble avoir été atteint puisqu'en 1822 on a pu, à l'aide de grappins, tirer la conduite jusqu'à la berge sans rompre la majorité des assemblages. Parmi les diverses mesures prises une seule pourrait paraître discutable : la grande épaisseur donnée aux parois ne procure pas tout l'avantage escompté, car les efforts nécessaires pour la mise en place du tuyau sont proportionnels à son poids ; finalement, la contrainte subie par le métal lorsque la conduite n'est soutenue que de distance en distance par des engins de levage n'est pas diminuée par cet accroissement d'épaisseur. Cette grande épaisseur reste avantageuse cependant pour résister aux accrochages et aux chocs subis au fond de l'eau (ancres de bateau, épaves glissant sur le fond), diminuant les risques de perforation, d'écrasement ou de déplacement de la canalisation.

\section{TOLÉRANCES ET PRÉCISION DANS LA FABRICATION}

L'étude des dimensions relevées nous montre que C. Cantius Pothinus a produit des tuyaux qui n'avaient pas tous exactement le même diamètre ou, plus exactement, le même périmètre, cette mesure étant plus significative puisque la section de ces tuyaux n'est pas parfaitement circulaire.

Cette dispersion des dimensions résulte de la combinaison de plusieurs causes :

- les feuilles de métal employées n'avaient pas toutes exactement la même largeur;

- lors de leur cintrage elles étaient plus ou moins bien appliquées sur le mandrin utilisé;

- les manipulations nécessaires entre la mise en forme et la soudure faisaient sans doute que, lors de la soudure, les bords de la feuille étaient plus ou moins jointifs.

Il nous est impossible d'évaluer séparément l'influence de ces divers facteurs, nous ne pouvons que 
constater le résultat global des trois premiers temps de la fabrication : coulée des feuilles, mise en forme et soudure.

Nous pouvons comparer entre elles soit les mesures du périmètre extérieur total des divers éléments (somme des mesures des colonnes $\mathrm{E}$ et $\mathrm{F}$ du tableau I), soit les dimensions des sections circulaires de même périmètre extérieur (tabl. I : colonnes $\mathrm{P}$ et $Q$, calculées à partir du périmètre mesuré) qui nous ont servi d'intermédiaires pour le calcul des épaisseurs de paroi à partir des poids relevés. Le résultat est évidemment le même, les valeurs extrêmes s'écartent d'un peu moins de $10 \%$ de la moyenne obtenue à partir des trente-trois fragments conservés que nous admettons représentative des dimensions demandées par le maître d'œuvre.

L'épaisseur des feuilles, déduite des poids des tuyaux (colonne $\mathrm{R}$ ), est beaucoup moins régulière, elle se situe dans une fourchette de plus ou moins $18 \%$ de la valeur moyenne.

Du point de vue de la régularité dimensionnelle, nous ne comparerons pas les produits de C. Cantius Pothinus à ceux de son collègue lyonnais anonyme, car nous n'avons pas, à Lyon, un échantillon suffisamment nombreux pour permettre une estimation valable des tolérances admises. Nous remarquons simplement que l'artisan avait plus de peine à mâ̂triser l'épaisseur des feuilles produites que leur largeur. Cela n'a rien de surprenant, il est facile de réaliser des moules de largeurs presque identiques si on les établit à l'aide d'un même outillage, par exemple un gabarit glissant sur des règles. Il est par contre impossible de mesurer une épaisseur au moment de la coulée car cette opération doit être conduite rapidement et sans interruption pour obtenir un métal homogène. Le procédé le plus simple pour obtenir des feuilles d'épaisseur déterminée est de contrôler la quantité de métal introduite dans le moule (poids proportionncl à la surfacc de la fcuille ct à l'ćpaisscur désirée) en fondant dans un four la quantité de métal correspondant à un tuyau (majorée du poids de métal nécessaire aux chenaux de coulée qui, eux aussi, peuvent avoir des dimensions assez régulières). La grande diversité des résultats obtenus suggère une question : l'artisan qui a fabriqué les tuyaux possédait-il une balance pour peser le métal qu'il préparait pour la coulée? Les variations d'épaisseur ne résulteraient-elles pas d'une appréciation «au jugé » de la quantité de métal mise en œuvre pour chaque tuyau? Si cette hypothèse est exacte, nous aurions là un indice de la pauvreté de l'outillage de l'artisan plombier.
Les tuyaux qui portent une inscription pondérale sont tous d'un poids par mètre inférieur à la moyenne, sept d'entre eux figurent parmi les neuf plus légers. Cela nous inciterait à penser que la pesée des tuyaux terminés, avec inscription du poids sur les objets, était une opération de contrôle à la réception des tuyaux par le responsable du chantier. On n'y aurait soumis que les éléments inspirant un doute, par exemple ceux qui, après une mesure avec un compas d'épaisseur forcément effectuée près des extrémités, auraient montré une épaisseur voisine du minimum exigé, la pesée permettant d'apprécier l'épaisseur moyenne de tout l'élément.

\section{UNITÉS DE LONGUEUR ET DE POIDS}

Les mesures des éléments conservés nous permettent d'évaluer les unités employées par les constructeurs.

\section{Unité de longueur}

La colonne D du tableau I donne la distance séparant les parties les plus larges des nœuds de soudure, pour les pièces comportant un élément complet avec les deux assemblages d'extrémités. Cette mesure est un peu plus courte que la longueur d'un élément de tuyau, en raison de l'emboîtement de 3 à $4 \mathrm{~cm}$ existant à chaque assemblage.

La valeur moyenne de $294,5 \mathrm{~cm}$ conduit à supposer des éléments de $298 \mathrm{~cm}$ environ, ce qui correspond bien à l'indication de Vitruve parlant de sections de tuyau de dix pieds de long. La valeur du pied est ici de $29,8 \mathrm{~cm}$. Les variations autour de la moyenne s'expliquent par le fait que l'olive de soudure n'est pas disposée de manière absolument régulière sur l'assemblage et par la précision limitée des mesures effectuées. Il se pourrait que la valeur réelle du pied employé soit un peu plus forte, car notre tuyau le plus court ( $\left.\mathrm{n}^{\circ} 30\right)$, qui s'écarte aussi assez fortement de la moyenne pour l'estimation de la valeur de la livre - comme nous le verrons au paragraphe suivant - pourrait avoir été un peu raccourci pour des impératifs de montage (par exemple, relier deux portions de conduites déjà assemblées, ou bien recommencer un assemblage raté...). En reprenant le calcul avec nos sept autres éléments, nous trouvons un pied de $29,9 \mathrm{~cm}$.

Nous devons donc admettre, pour la conduite de notre chantier l'usage d'un pied d'une trentaine de centimètres, valeur proche des $29,6 \mathrm{~cm}$ communément admis pour la valeur du pied romain ${ }^{5}$.

5 Voir, par exemple, F. Kretzschmer, La technique romaine, Bruxelles, 1966, p. 10. 
Tableau III - Calcul de la valeur de la livre en accord avec les inscriptions numériques incisées sur certains éléments. Les deux dernières lignes sont la reprise du calcul pour les fragments 12 et 30 en les supposant raccourcis l'un avant, l'autre après la pesée de réception (élimination d'un défaut situé près d'une extrémité, nécessité de montage de la canalisation).

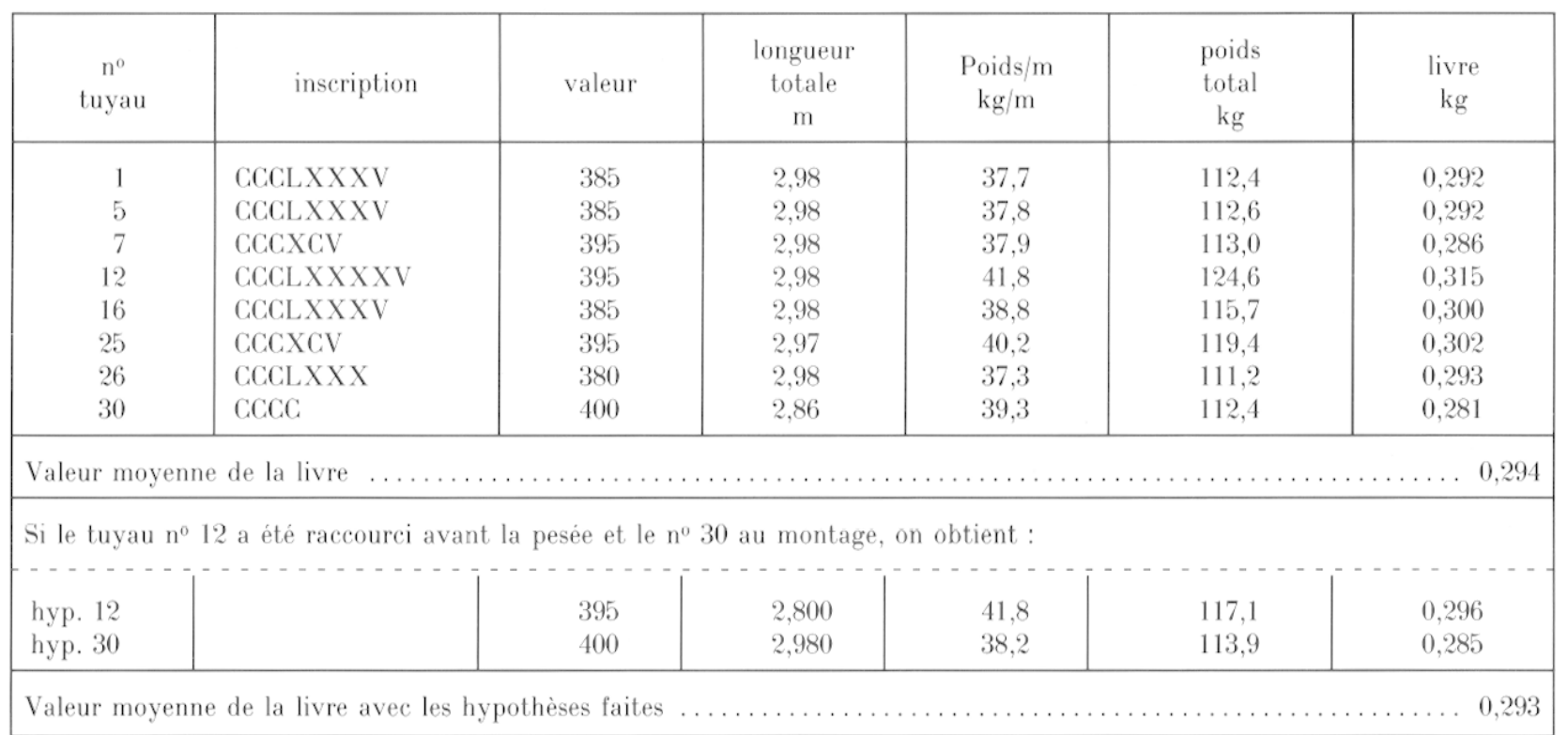

Cette valeur du pied donne une mesure tout à fait plausible de 1 pied 2/16 (un pied et deux doigts) pour la largeur des feuilles de plomb employées à la fabrication de nos tuyaux.

Le diamètre intérieur moyen de $9,4 \mathrm{~cm}$ (tabl. I, colonne Q) correspond à la dimension des vicenaria de Frontin ${ }^{6}$.

\section{Unité de poids}

A partir des huit tuyaux marqués de CCCLXXX à CCCC nous pouvons essayer de déterminer la valeur de l'unité de poids utilisée. Ce calcul permet en outre de vérifier le bien fondé de l'interprétation de ces inscriptions en tant qu'indications pondérales (tabl. III). Si cette interprétation est correcte, les divers objets doivent renvoyer à une mème unité.

Comme pour l'évaluation de l'épaisseur moyenne, nous avons déduit du poids actuel de chaque élément le poids estimé des masses de soudure. Pour les éléments 25 et 30 conservés en entier nous n'avons eu qu'à diviser ce poids par la valeur

6 Discuter l'interprétation des mesures données par Frontin (Les aqueducs de la ville de Rome, Paris, Les Belles Lettres, 1961) comme normalisées pour le réseau de distribution d'eau de Rome déborderait le cadre du présent article. Un tableau traduisant ces divers calibres en mesures modernes se trouve dans Kretzschmer, op. cit. p. 55). indiquée pour obtenir une évaluation de l'unité employée. Pour les autres éléments, conservés seulement en partie, nous avons rétabli, par une règle de trois, le poids d'un élément standard de dix pieds. Les résultats obtenus donnent une valeur moyenne de $294 \mathrm{~g}$, avec des valeurs extrêmes de 281 et $315 \mathrm{~g}$.

Les constructeurs n'ont donc pas employé la livre romaine dont la valeur est estimée de 325 à $327 \mathrm{~g}$, selon les divers auteurs ?.

La dispersion de nos évaluations pourrait être réduite si pour le tuyau $n^{\circ} 30$ qui nous donne la valeur la plus faible nous retenons l'hypothèse indiquée précédemment, soit un tuyau de dix pieds lors de sa réception et quelque peu raccourci lors du montage. En lui prêtant une mesure de dix pieds ou $2,98 \mathrm{~m}$ lors de sa pesée, il correspondrait à une livre de $293 \mathrm{~g}$. De même le numéro 12 entrerait lui aussi dans la fourchette $285-300 \mathrm{~g}$ si on a accepté à la réception un élément un peu plus court que les dix pieds réglementaires (par exemple, raccourci à 2,80 m par le fabricant pour éliminer un défaut situé à une extrémité). Notre valeur moyenne de $294 \mathrm{~g}$ est elle-même entachée d'une certaine incertitude. Nos mesures de longueurs et de poids comportant une certaine imprécision (longueurs des éléments à $1 \mathrm{~cm}$

7 Pour l'estimation de la livre : DAREMBERg et SAglio, Dictionnaire des Antiquités, article LIBRA. 
près, dimensions des cordons de soudure à 0,1 ou $0,2 \mathrm{~cm}$ près, poids à 0,2 ou $0,3 \mathrm{~kg}$ près) et notre évaluation du poids des assemblages, basée sur leurs dimensions moyennes et une densité de la soudure de $10 \mathrm{~g} / \mathrm{cm}^{3}$ adoptée dans l'ignorance de la densité exacte de l'alliage plomb-étain, entraînent une possibilité d'erreur approchant les $5 \%$ dans le cas où toutes les erreurs s'accumuleraient et joueraient dans le même sens pour les huit objets étudiés. Les pesées effectuées dans l'Antiquité n'étaient sûrement pas d'une extrême précision, les résultats n'ont été exprimés que sous une forme arrondie à cinq livres près. Il nous faut donc nous contenter de conclure à l'emploi d'une unité de poids comprise entre 280 et $310 \mathrm{~g}$, une valeur comprise entre 290 et $300 \mathrm{~g}$ étant extrêmement probable, d'après la répartition de nos huit estimations.

Il peut s'agir d'une mesure locale et il serait intéressant de rechercher s'il existe d'autres indices de l'emploi de cette unité.

\section{CONDUITE DU CHANTIER}

Nous n'envisageons que l'hypothèse d'une conduite immergée et nous excluons celle d'une canalisation placée sur un pont fixe ou mobile (pont de bateaux) tombée au fond du fleuve à la suite de la ruine de l'ouvrage porteur.

En effet, une conduite située sur un pont rigide n'exige pas les précautions exceptionnelles prises dès la fabrication des tuyaux et lors de leur montage pour obtenir le plus de solidité possible, les réparations éventuelles ne présentant pas de difficultés parliculières. Il semble d'ailleurs qu'il n'y ait pas eu un tel pont à Arles dans l'Antiquité. Sur un pont de bateaux, la conduite de plomb n'aurait pu subir sans accident les déformations rapides dues à l'enfoncement variable des flotteurs sous le poids des charges mobiles ni celles plus importantes nécessaires à chaque extrémité du tablier pour suivre les variations de hauteur des eaux du fleuve.

La méthode d'installation la plus simple est celle du montage de la canalisation sur le tablier d'un pont de bateaux qui a existé entre Arles et
Trinquetaille, ou d'un ouvrage provisoire établi pour la durée du chantier, peut-être en imposant certaines restrictions à la circulation des charges lourdes sur l'ouvrage pendant la fin du montage. Dans un deuxième temps, la conduite aura été un peu soulevée à l'aide de chèvres placées sur le bord du pont, poussée latéralement de quelques décimètres, et immergée en relâchant, de manière coordonnée, les cordages de suspension, jusqu'à ce que leur "mou" garantisse l'appui sur le fond.

Le montage de la conduite n'a pas dû présenter de difficultés particulières, mais nous pouvons remarquer que les emboîtements des éléments les uns dans les autres n'ont pas tous été effectués dans le même sens (fig. 15). Huit des tuyaux tels qu'ils sont conservés (n ${ }^{\circ s} 9,10,14,19,21,23,25,30$ ) ont gardé tout ou partic des asscmblages de chacune de leurs extrémités : sept d'entre eux sont évasés à une extrémité et dépouillés du cordon de soudure longitudinal à l'autre extrémité pour pouvoir s'emboîter dans l'évasement de l'élément adjacent, ils ont donc une extrémité mâle et une femelle. L'un d'eux ( $\left.n^{\circ} 25\right)$ est évasé à ses deux extrémités. Il y avait donc des emboittements disposés de manière différente par rapport à la direction d'écoulement de l'eau.

Nous expliquerions volontiers cette particularité en considérant l'élément 25 comme le premier posé de la conduite, à peu près en son milieu, le montage progressant à partir de ce point central vers les deux rives, ce qui permet de faire travailler simultanément deux équipes de monteurs, et donc de doubler la vitesse de montage par rapport à une construction avançant d'une rive vers l'autre.

La mise en place de la conduite au fond du Rhône a constitué la phase la plus délicate de l'entreprise. La difficulté de cette opération provient de la très faible résistance du plomb à la traction, entraînant une très faible résistance du tuyau à la flexion. Il est difficile de se livrer à des calculs précis de résistance des matériaux puisque les modules d'élaslicilé du plomb à la traction et à la compression sont très différents, que les résistances mesurées sur des éprouvettes de plomb dépendent énormément de la vitesse d'application de la charge et que

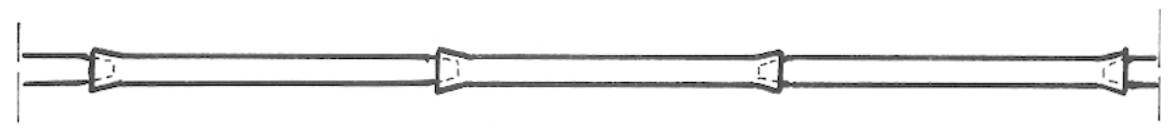

Fig. 15 - Les assemblages ont été réalisés en disposant les emboitements dans les deux directions opposées à partir de l'élément central. 
la résistance des assemblages semble encore plus difficile à évaluer que celle du tuyau.

Les divers calculs approximatifs auquels nous nous sommes livrés semblent indiquer qu'il est impossible d'admettre une portée de plus de $2 \mathrm{~m}$ entre les appuis consécutifs lors de la manipulation de la canalisation; cela correspond aussi à l'expérience acquise par la manutention des éléments conservés : un élément d'environ $3 \mathrm{~m}$ soutenu seulement en son milieu pendant la pesée sur la bascule se courbe (flèche de près de $10 \mathrm{~cm}$ ); porté en le soutenant en deux points distants d'environ 1,50 m, sa flèche est encore visible à l'œil nu.

Il est donc vraisemblable que la canalisation a été suspendue au moins en deux points pour chaque élément de dix pieds. Même si un système de palonniers permet de n'établir qu'une chèvre pour dcux ou quatre de ces points de suspension, il a fallu une main d'œuvre assez nombreuse et disciplinée pour assurer la manœuvre simultanée, bien synchronisée de cinquante à cent treuils, avec un code de signaux très précis, les points d'appui successifs ne devant jamais avoir de différence de hauteurs de plus de 3 ou $4 \mathrm{~cm}$.
Mais cette difficulté n'est pas la seule, toutes ces précautions sont inutiles si, une fois immergée, la conduite repose mal sur le fond; le tuyau n'est que très peu soulagé de son propre poids par la poussée d'Archimède qui ne représente que le quart du poids du tuyau vide. Si, par suite d'inégalités du fond, une portion de quelques mètres ne porte pas sur le sol, la rupture de cette partie fléchie est probable. Pour éliminer ce risque le constructeur a donc dû procéder avant même l'ouverture du chantier, par une série de sondages, à un relevé du profil transversal du lit du fleuve selon le tracé prévu, pour s'assurer que le fond présente une courbe régulière, sans creux ou bosses prononcées. Il est très probable aussi que ce relevé a servi pour donner à la conduite une courbure correspondant à celle du fond (et même un peu plus prononcée) afin qu'au moment de son immersion elle commence à porter d'abord au milieu de la traversée et que le contact avec le fond s'établisse progressivement à partir de ce point central en jouant sur la déformation de quelques centimètres admissible entre les appuis consécutifs (fig. 16 et 17). Sans cette précaution, la conduite porterait d'abord près des rives, dans les zones peu profondes du fleuve, et la

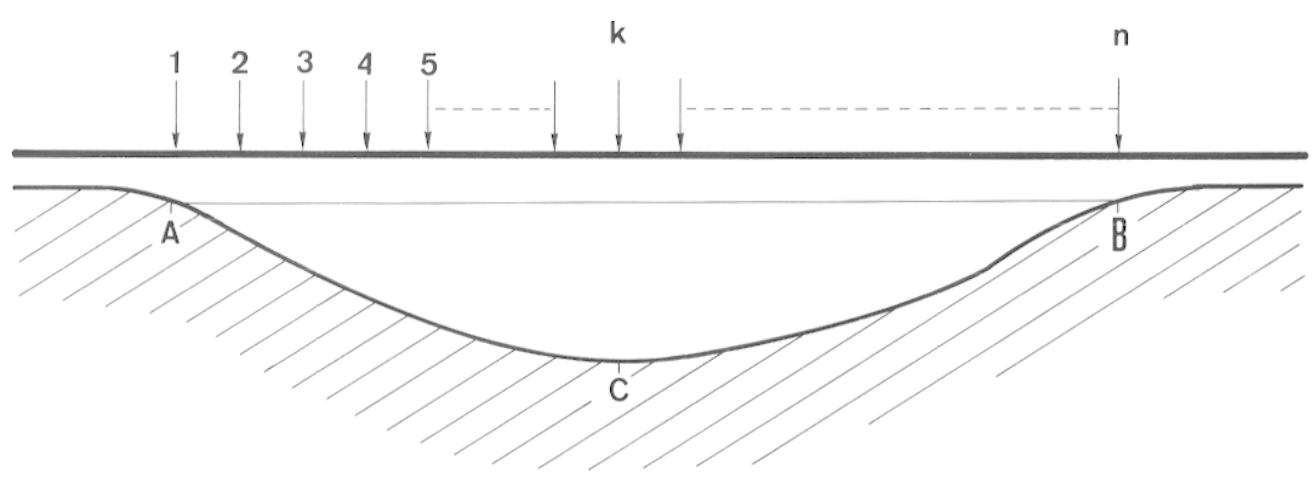

Fig. 16 - Représentation schématique de la canalisation avant que ne commence son immersion. Les flèches $1,2,3 \ldots \mathrm{n}$ suggèrent les points de suspension.

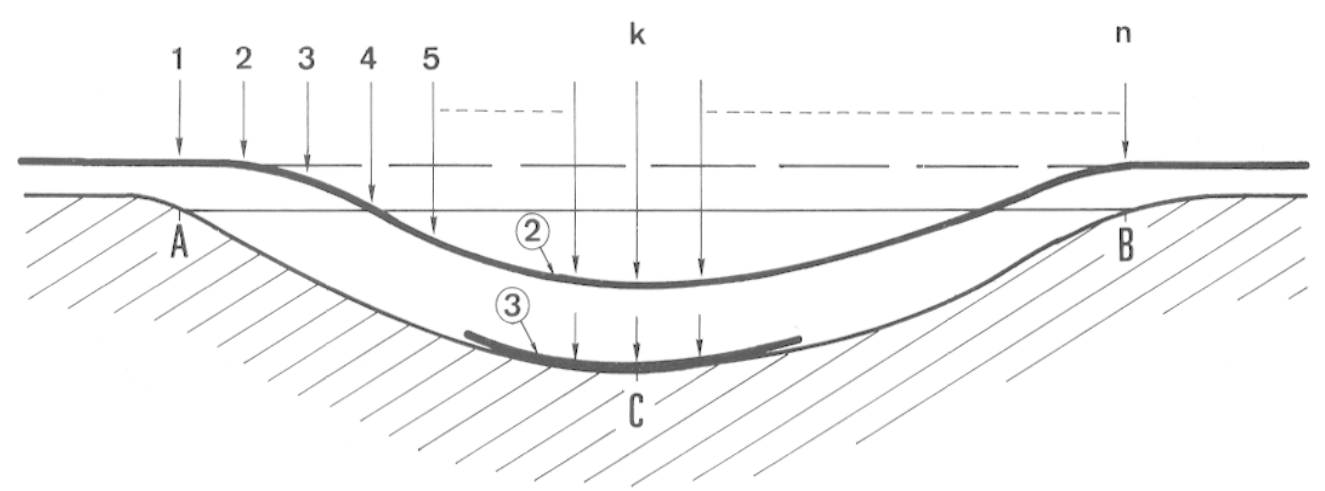

Fig. 17 - Représentation schématique de l'immersion de la conduite, en la courbant peu à peu pour lui donner une forme correspondant au profil du lit du fleuve, le contact avec le fond s'élablissant d'abord au point $\mathrm{C}$, le plus profond.

Les lignes 2 et 3 représentent des étapes intermédiaires. 
portion centrale, rectiligne, devrait épouser les inflexions d'une courbe, plus longue qu'elle, ce qui entraînerait une traction sur les parties reposant déjà sur le fond, traction risquant de provoquer une ruplure, par déboîtement d'un assemblage, le frottement des tuyaux sur le sol s'opposant au glissement vers le milieu du fleuve. Dans le cas où le contact est établi d'abord au milieu de la traversée et progresse vers les rives, les extrémités de la conduite peuvent se déplacer bien plus librement car elles sont suspendues à des câbles souples.

On peut aussi imaginer la pose se déroulant à partir d'une rive, la conduite recevant une courbure calculée pour demeurer admissible, le contact avec le fond s'établit alors en progressant d'une rive vers l'autre. Dans cette hypothèse là encore faut-il être certain que le fond présente une surface régulière, sans saillies ou creux prononcés, si on veut éviter de laisser une partie de la canalisation privée d'appui entre deux bosses.

Une fois la conduite entrée dans l'eau, dans l'un ou l'autre cas, la mancuvre de descente doit se faire en mesurant la longueur de câble filée par chaque treuil, pour être certain de ne pas créer localement une courbure trop accentuée qui serait sanctionnée par une rupture ruineuse, ou une fissure dans un noud de soudure, provoquant la fuite dans le Rhône de l'eau transitant par la canalisation.

Aucune de ces opérations ne nécessite de connaissances théoriques exceptionnelles : tous les problèmes géométriques peuvent se résoudre graphiquement, en traçant sur le sol, en grandeur réelle, le profil du fond pour déterminer les mouvements à opérer à chaque point de suspension à chaque phase de l'opération. Pour diriger cette main d'œuvre nombreuse (au moins cent à deux cents hommes aux treuils et d'une centaine de manœuvres pour écarter la conduite des barges portant le pont et contrôler ses mouvements transversaux), il a fallu toute une équipe de surveillants et la direction d'un bon géomètre, sûr de ses levées et des mesures prises sur ses épures, doté d'un bon coup d'œil pour suivre l'ensemble d'un mouvement pour lequel le droit à l'er- reur est exclu. Il a fallu aussi un système de signaux pour indiquer avec précision les mouvements à effectuer.

Cette opération d'immersion a certainement eu lieu en période d'étiage, pour diminuer les aléas dus au courant et réduire la longueur de la portion immergée pour laquelle toute rupture équivaut à la perte de l'ouvrage. S'il existait des rives basses, submergées seulement en période de hautes eaux, il était plus facile d'y travailler à sec en profitant des étiages que d'y immerger la conduite.

L'immersion a dû être conduite fort lentement, pour qu'on puisse vérifier à chaque treuil la longueur de câble filé et la bonne coordination avec les treuils voisins : il est donc probable qu'à conditions égales de courant et de débit du Rhône, le constructeur ait préféré les longues journées d'été à la saison d'hivẹr. C'est sans doute pouce par pouce qu'on a opéré, il n'est pas impossible que cette phase critique du chantier ait duré plus d'une journée.

Les Arlésiens d'alors ont élé témoins d'une prouesse technique, fondée avant tout sur la discipline des exécutants.

André Cochet

Nola Bene

C'est grâce à l'accueil bienveillant que M. Rouquette, conservateur du musée Réatlu, nous a ménagé que cette étude a pu être menée à bien.

Le personnel du musée nous a fourni une aide particulièrement précieuse pour la manutention d'objets à la fois lourds et fragiles qu'il a fallu retirer des réserves, déplacer pour examen, photographie, pesée... avant de les ranger, au total $3925 \mathrm{~kg}$ déplacés à plusieurs reprises.

Je remercie également la direction et le personnel de laboratoire de la société Métaleurop.

Enfin, j'exprime une gratitude toute particulière à mon ami J. Hansen. C'est lui qui le premier a attiré mon attention sur le caractère exceptionnel de la canalisation Arles-Trinquetaille. Nous en avons entrepris l'étude ensemble. Des raisons d'ordre professionnel l'ont privé du temps qui lui aurait été nécessaire pour étudier les données recueillies et participer à la mise en forme de la publication. 


\section{ANNEXE I}

\section{Histoire de la découverte}

"Le 4 du mois de juin 1822, le capitaine du bateau Le Hardi (Pierre Jourdan), de la ville d'Arles, obligé de jeter son ancre au milieu du Rhône, vers la pointe de la Camargue, l'accrocha contre une suite de tuyaux de plomb que, le lendemain, à l'aide de quelques marins, il traîna jusque sous la voûte de la porte dite de Rousset, après en avoir laissé dans le fleuve un reste assez important qui se détacha de la partie dont il s'était rendu maitre ...".

Ainsi débute la "notice sur d'anciens tuyaux de plomb trouvés à Arles, adressée à la Société royale des Antiquaires de France, par M. le comte de Villeneuve-Bargemont, préfet du département des Bouches du Rhône, correspondant" dans les Mémoires de la Société..., 1824, p. 232-246.

Cette note paraît être la source que les auteurs postérieurs ont largement exploitée. A l'occasion de la découverte de 1822, l'auteur rappelle et commente les trouvailles antérieures et semble avoir consulté personnellement les diverses sources manuscrites qu'il cite assez abondamment.

La partie demeurée dans le fleuve sera retirée "quelques années plus tard" comme l'indique L.-A. Constans, en 1921, dans son ouvrage sur Arles antique, p. 400.

La découverte de 1822 est située avec précision : la conduite, orientée est-ouest, traversait le Rhône entre un point de la rive droite situé à $115 \mathrm{~m}$ en amont du débouché du chemin du cimetière de Trinquetaille sur la chaussée de la berge et un point de la rive gauche situé à $250 \mathrm{~m}$ en amont de l'écluse de la roubine du Roi (distances mesurées en suivant la berge du fleuve).

La canalisation aurait donc èté placée à environ $60 \mathrm{~m}$ en aval du pont du chemin de fer et 200 à $250 \mathrm{~m}$ en amont du pont de bateaux existant dans l'Antiquité qui aboutissait, sur la rive gauche, en face de la rue de l'Amphithéâtre (op. cit., p. 341). Si celle lopographie est exacte le chantier a exigé l'établissement d'un pont de bateaux provisoire.
"La longueur totale de ces tuyaux (ceux retirés le 5 juin 1822) est de 39 pieds et demi. Chacun de ceux qui sont entiers a neuf pieds trois pouces. La circonférence est de treize pouces et demi; l'épaisseur du plomb est de cinq lignes.

Le poids de chacun de ces tuyaux est buriné sur la soudure du dos; les deux bouts qui ne sont point dans leur entier, ne le portent pas. On lit sur le premier CCCCX, sur le deuxième CCCLXXXV, sur le troisième $\mathrm{CCCXCV}$, et sur le quatrième CCCLXXXV».

Les dimensions indiquées correspondent à celles des tuyaux que nous avons étudiés; notre collection actuelle est beaucoup plus importante et il se peut qu'elle comporte quelques échantillons des trouvailles antérieures, sans doute aussi la partie abandonnée au fond et repêchée ultérieurement. Aucun des éléments conservés ne portant l'indication pondérale CCCCX, il est certain qu'au moins un des tuyaux découverts en juin 1822 a disparu.

Une première découverte connue est attribuée à l'année 1570 , par M. Rebattu, antiquaire d'Arles, de la fin du xvile s. Une autre, plus précisément décrite, a eu lieu le 26 avril 1707, vis à vis de la pointe de la Camargue, à la suite de l'accrochage de l'ancre d'un bateau : "On en retira de l'eau une grande quantité (de tuyaux de plomb) qui étaient emmanchés les uns dans les autres, et très bien soudés; ayant chacun dix pieds de longueur d'une emmanchure à l'autre ; treize pouces de circonférence, quatre pouces deux tiers de diamètre et un demi-pouce d'épaisseur; que sur chaque tuyau, il était écrit en caractères d'un pouce de hauteur: C. CANTIVS. POIHINVS. FAC.». Cette description sommaire correspondrait bien à une section de la canalisation trouvée en 1822 .

Les tuyaux retirés du fleuve en 1570 auraient porté en plus de la marque C. CANTIVS. POINVS. FAC l'inscription T. VAL. S. GAL. MAE LE. Le fait demeure cependant douteux. M. Rebattu écrivait 
plus d'un siècle après la trouvaille et des confusions sont possibles car les découvertes de tuyaux de plomb ont été nombreuses à Arles; l'information gagnerait à être confirmée par des témoignages plus directs ou de nouvelles découvertes.

Notons que les découvertes de 1650 et des années environnantes, faites dans les maisons des sieurs "Claude Raspal et $\mathrm{M}^{\mathrm{r}}$ Tourré" portaient sur des tuyaux de grandes dimensions, "chaque pièce pesait plus de quinze quintaux sur deux toises de longueur" et un élément portait l'inscription DLXXXIII. Cinq cent quatre vingt trois livres de $295 \mathrm{~g}$ auraient correspondu à un poids de $172 \mathrm{~kg}$ pour un élément de dix pieds, soit environ $58 \mathrm{~kg}$ par mètre de canalisation. D'après ce que nous avons observé sur d'autres sites, pour les tuyaux à paroi relativement mince d'usage courant, cela correspondrait à des canalisations d'un périmètre de 45 à
$50 \mathrm{~cm}$. Il semble que ces tuyaux ne nous soient pas parvenus; nous n'avons rien vu de ce genre dans les collections du musée.

M. de Villeneuve-Bargemont précise aussi l'emplacement origincl du tombeau de Quintus Candidus Begninus : il se trouvait à Trinquetaille «à la pointe de la Camargue, peut-être même sur la direction des canaux de plomb du Rhône" avant d'être transporté en Camargue, au domaine de M. Eyminy. Cette proximité ne prouve pas que $Q$. Candidus Begninus soit le constructeur de la canalisation sous-fluviale d'Arles à Trinquetaille, mais explique peut-être pourquoi l'hypothèse s'est imposée à l'esprit de certains auteurs. Nous nous garderons de prendre parti dans les discussions suscitées par la traduction et l'interprétation de cette épitaphe.

A.C.

\section{ANNEXE II}

\section{Description de la marque}

Les tuyaux découverts au fond du Rhône et le fragment conservé au musée de Nîmes portent une marque en relief, obtenue par moulage lors de la coulée de la feuille de plomb destinée à la fabrication des tuyaux.

Chacun des éléments porte soit une, soit deux marques, disposées de manière à être lues normalement lorsque le tuyau est disposé le cordon de soudure placé en haut, position ordinaire des canalisations romaines. Lorsqu'il y a deux marques, elles sont placées sensiblement en regard l'une de l'autre, de part et d'autre du cordon de soudure.

Toutes ces marques sont identiques, sauf pour le relief qui est plus ou moins accusé selon les exemplaires. Elles ont été moulées à l'aide d'une petite plaque modèle, portant l'inscription en relief. On voit nettement sur l'exemplaire nîmois, comme sur l'un ou l'autre des fragments arlésiens, une partie du contour rectangulaire de cette petite plaque modèle. C'est l'impression plus ou moins énergique de cet outil sur le sable du moule qui est à l'origine de la diversité dans le relief des lettres.

On lit aisément le texte de l'inscription :

C. CANTIVS. POIHINVS. FAC
Le premier point est disposé à l'intérieur du $\mathrm{C}$ initial, les deux ponctuations consistent en une feuille disposée pointe vers le bas et il manque la barre horizontale du $\mathrm{T}$ de Pothinus.

Dimensions :

- longueur totale du texte : $32,5 \mathrm{~cm}$;

- hauteur des lettres : 2,8 à $3 \mathrm{~cm}$;

- la saillie des caractères par rapport à la surface des feuilles de plomb est en général de 1 ou $2 \mathrm{~mm}$.

Par analogie avec les nombreuses marques de ce genre portées par des tuyaux de plomb de diverses villes nous proposons d'y voir la marque du producteur du tuyau (nom au nominatif, FAC développé en facit) :

\section{CANTIVS. POTHINVS. FACit}

Le fait que cette marque apparaisse sur le fragment conservé à Nîmes, très certainement étranger à la canalisation Arles-Trinquetaille, incite à voir en $\mathrm{C}$. Cantius Pothinus le plombier producteur des tuyaux plutôt que le maitre d'œuvre de la canalisation sousfluviale. 


\section{ANNEXE III}

\section{Analyses chimiques}

Nous devons à l'obligeante collaboration de la société Metaleurop l'analyse chimique des tuyaux $\mathrm{n}^{\text {os }} 1$ à 14 . Le $\mathrm{n}^{\circ} 10$ a fait l'objet de deux prélèvements, à chacune des extrémités, afin de permettre d'apprécier l'homogénéité du métal d'un même tuyau.

Ces analyses ont été effectuées par le laboratoire de l'usine de Noyelles-Godault selon le procédé I.C.P. (spectrographie d'émission, les échantillons mis en solution sont injectés dans une torche à plasma).

Ayant pu faire analyser à plusieurs reprises des échantillons tirés d'un même objet par ce même laboratoire selon ce même procédé et aussi par spectrographie d'émission par étincelage, il semble que les résultats obtenus soient tout à fait reproductibles. Pour l'argent, le bismuth, le cuivre, les valeurs indiquées doivent comporter une marge d'incertitude d'environ $10 \%$ en plus ou en moins, malgré les très faibles teneurs dosées. Le tableau IV donne les résultats de ces analyses.

On constate qu'il s'agit d'un métal très pur, d'une pureté comparable à celle du plomb doux affiné mis sur le marché dans les années 1920, avec seulement un peu plus d'argent.

La grande pureté du métal employé et la complexité de la métallurgie du plomb interdisent de remonter de la composition chimique du métal à l'identification d'un minerai.

Voici à ce sujet l'opinion des ingénieurs métallurgistes de Noyelles-Godault :

"Toutes les analyses réalisées montrent que les impuretés, sauf peut-être l'étain, restent à des niveaux très bas : tous ces échantillons proviennent de plomb purifié (plomb doux) et non d'alliage de plomb (...).
Les impuretés cuivre, argent, arsenic, zinc et cadmium sont à des niveaux bas et correspondent à des impuretés éliminées par simple oxydation lente à l'air (As, $\mathrm{Zn}, \mathrm{Cd}$ ) ou par refroidissement et cristallisations successives $(\mathrm{Ag}, \mathrm{Cu})$.

Ces niveaux d'impureté ne dépendent pas des teneurs initiales dans les minerais, mais dépendent du type et du nombre des traitements affinant le métal. Ces impuretés ne peuvent pas caractériser la nature du minerai utilisé.

La seule impureté insensible au traitement par affinage est le bismuth. Les teneurs anormalement basses en bismuth peuvent s'expliquer soit par un

Tableau IV - Analyses chimiques : la première colonne donne le numéro des objets. Toutes les teneurs sont en partie par million (ppm). La colonne de droite indique la somme de toutes les impuretés dosées.

\begin{tabular}{|r|r|r|r|r|r|r|r|r|r|r|r|r|r|r|}
\hline $\begin{array}{c}\text {. } \\
\text { objet }\end{array}$ & $\mathrm{Ag}$ & $\mathrm{Bi}$ & $\mathrm{Ni}$ & $\mathrm{Cu}$ & $\mathrm{Cd}$ & $\mathrm{Te}$ & $\mathrm{Zn}$ & $\mathrm{Se}$ & $\mathrm{As}$ & $\mathrm{TI}$ & $\mathrm{Sn}$ & $\mathrm{Sb}$ & $\mathrm{S}$ & total \\
\hline $\mathrm{l}$ & 27 & 20 & 10 & 180 & 5 & 20 & 5 & 20 & 20 & 20 & 250 & 50 & 60 & 687 \\
2 & 23 & 20 & 10 & 250 & 5 & 20 & 5 & 20 & 20 & 20 & 1465 & 80 & 50 & 1988 \\
3 & 24 & 20 & 10 & 160 & 5 & 20 & 85 & 20 & 20 & 20 & 250 & 60 & 50 & 744 \\
4 & 47 & 20 & 10 & 160 & 5 & 20 & 5 & 20 & 20 & 20 & 500 & 75 & 60 & 962 \\
5 & 41 & 20 & 10 & 155 & 5 & 20 & 5 & 20 & 20 & 20 & 670 & 60 & 50 & 1096 \\
6 & 22 & 20 & 15 & 200 & 5 & 20 & 24 & 20 & 20 & 29 & 1370 & 80 & 60 & 1885 \\
7 & 35 & 20 & 10 & 220 & 5 & 20 & 5 & 20 & 20 & 39 & 2375 & 65 & 50 & 2884 \\
8 & 39 & 20 & 14 & 250 & 5 & 20 & 35 & 20 & 20 & 20 & 290 & 80 & 50 & 863 \\
9 & 19 & 20 & 13 & 160 & 5 & 20 & 5 & 20 & 20 & 20 & 10 & 60 & 50 & 422 \\
10 & 18 & 20 & 20 & 190 & 5 & 20 & 5 & 20 & 20 & 20 & 10 & 60 & 60 & 468 \\
10 & 19 & 20 & 20 & 190 & 5 & 20 & 5 & 20 & 25 & 24 & 10 & 100 & 200 & 658 \\
11 & 37 & 20 & 12 & 195 & 5 & 20 & 5 & 20 & 30 & 21 & 360 & 60 & 60 & 845 \\
12 & 46 & 20 & 10 & 170 & 5 & 20 & 5 & 20 & 20 & 20 & 370 & 40 & 70 & 816 \\
13 & 44 & 20 & 12 & 250 & 5 & 20 & 5 & 20 & 25 & 25 & 1800 & 120 & 80 & 2426 \\
14 & 41 & 20 & 10 & 230 & 5 & 20 & 5 & 20 & 30 & 20 & 1620 & 120 & 130 & 2271 \\
\hline
\end{tabular}


niveau de bismuth dans le minerai extrêmement bas, soit par une sélection d'oxydes à basse teneur en bismuth lors de la coupellation, ce qui semble peu probable, car, dans ce cas les teneurs en cuivre et argent devraient être encore plus basscs" (lettre du 10 août 1989).

A cet avis - on ne peut plus autorisé - nous n'ajouterons que deux remarques.

1. L'étain n'est pas significatif, il s'élimine facilement par oxydation à l'affinage, mais il est présent dans l'atelier du plombier qui l'emploie pour les soudures. Il peut s'introduire dans le plomb par la refonte d'objets récupérés, comportant des soudures faites à l'aide d'alliages plomb-étain.

2. A propos de la question affinage thermique ou revivification de litharges issues de la coupella- tion, il ne faut pas oublier que les métallurgistes antiques opéraient dans des conditions très différentes de celles réalisées dans les usines modernes. Aujourd'hui, on effectue l'affinage sur des quantités considćrables de métal, au moins plusieurs dizaines de tonnes à la fois, le refroidissement d'une telle masse est très lent, les différentes phases qui se forment pendant la solidification peuvent aisément se séparer les unes des autres en raison de leurs différences de densité... Dans l'Antiquité, en travaillant sur de beaucoup plus petites quantités, l'affinage thermique devait être moins efficace et la possibilité de la revivification de litharges issues de la coupellation d'un plomb argentifère demeure peut-être ouverte.

A.C. 